\title{
Differential expression patterns of metastasis suppressor proteins in basal cell carcinoma
}

\author{
Onder Bozdogan ${ }^{\mathrm{I}, 2}$, MD, Isik G. Yulug ${ }^{\mathrm{I}}, \mathrm{PhD}$, Ibrahim Vargel $^{3}, \mathrm{MD}, \mathrm{PhD}$, \\ Tarik Cavusoglu ${ }^{4}$, MD, Ayse A. Karabulut ${ }^{5}$, MD, Gurbet Karahan ${ }^{\mathrm{I}}$, PhD Student, and \\ Nilufer Sayar ${ }^{\mathrm{I}}$, PhD Student
}

\begin{abstract}
${ }^{1}$ Department of Molecular Biology and Genetics, Faculty of Science, Bilkent University, Ankara, Turkey, ${ }^{2}$ Department of Pathology, Medical Faculty, Kırıkkale University, Kırıkkale, Turkey, ${ }^{3}$ Department of Plastic Surgery, Medical Faculty, Hacettepe University, Ankara, Turkey, ${ }^{4}$ Department of Plastic Surgery, Medical Faculty, Kırıkkale University, Kırıkkale, Turkey, and ${ }^{5}$ Department of Dermatology, Medical Faculty, Kırıkkale University, Kırıkkale, Turkey
\end{abstract}

\section{Correspondence}

Associate Prof. Isik G. Yulug, PhD Department of Molecular Biology and Genetics

Bilkent University Faculty of Science TR-06800, Ankara, Turkey

E-mail: yulug@fen.bilkent.edu.tr

Conflicts of interest: None.

\begin{abstract}
Background Basal cell carcinomas (BCCs) are common malignant skin tumors. Despite having a significant invasion capacity, they metastasize only rarely. Our aim in this study was to detect the expression patterns of the NM23-H1, NDRG1, E-cadherin, RHOGDI2, CD82/KAl1, MKK4, and AKAP12 metastasis suppressor proteins in BCCs.

Methods A total of $96 \mathrm{BCC}$ and 10 normal skin samples were included for the immunohistochemical study. Eleven frozen BCC samples were also studied by quantitative real time polymerase chain reaction (qRT-PCR) to detect the gene expression profile.

Results NM23-H1 was strongly and diffusely expressed in all types of BCC. Significant cytoplasmic expression of NDRG1 and E-cadherin was also detected. However, AKAP12 and CD82/KAl1 expression was significantly decreased. The expressions of the other proteins were somewhere between the two extremes. Similarly, qRT-PCR analysis showed down-regulation of AKAP12 and up-regulation of NM23-H1 and NDRG1 in BCC.

Morphologically aggressive BCCs showed significantly higher cytoplasmic NDRG1 expression scores and lower CD82/KAl1 scores than non-aggressive BCCs.

Conclusion The relatively preserved levels of NM23-H1, NDRG1, and E-cadherin proteins may have a positive effect on the non-metastasizing features of these tumors.
\end{abstract}

\section{Introduction}

Basal cell carcinoma of the skin (BCC), a common human carcinoma, tends to be locally invasive and metastasizes only rarely. ${ }^{\mathrm{I}}$ Despite the low metastasizing ability, these lesions show significant invasion capacity if neglected. ${ }^{2}$ This unusual predisposition makes BCC an interesting biological model for invasion and metastasis.

Metastasis is a complex biological process and is controlled by various mechanisms. One important mechanism is provided by metastasis suppressor proteins (MSPs). MSPs inhibit or suppress metastasis without any effect on cell proliferation and affect different steps of the complex metastasizing process. To date, more than 30 MSPs have been identified. ${ }^{3}$ In this study, we investigated the expression patterns of seven well-defined important MSPs, including NM23-HI, NDRGI, E-cadherin, RHOGDI2, CD82/KAII, MKK 4 , and AKAPI 2 in BCCs.
$\mathrm{NM}_{23}-\mathrm{HI}$ is the first described MSP and is downregulated in several metastatic cell lines and in a group of human carcinomas. ${ }^{4} \mathrm{NM} 23-\mathrm{H}_{I}$ gene encodes a nucleoside diphosphate kinase A. Although the metastasis suppressor mechanism of $\mathrm{NM} 23-\mathrm{HI}$ is not clear, its interaction with kinase suppressors of RAS and, as a result, alteration of the MAPK signaling pathway is a probable mechanism. ${ }^{3}$ It has also recently been suggested that it suppresses metastasis by inhibiting the expression of EDG2 (lysophosphatidic acid receptor). ${ }^{5}$

NDRGI (N-myc downstream regulated I) is a member of the NDRG family of proteins and has been shown to reduce metastasis in colon, breast, and prostate neoplasms. ${ }^{6}$ Although the metastasis suppressor mechanism of NDRGI is not clear, the interaction with the cell-cell adhesion molecules $\beta$-catenin and E-cadherin might be a possible mechanism. ${ }^{7,8}$

E-cadherin is a well-known cell-cell adhesion protein, and loss of its expression plays important roles in tumor 
invasion and metastasis. ${ }^{9, \text { IO }}$ It also functions as a negative regulator of the canonical WNT signaling pathway. E-cadherin has been extensively studied in human tumors, including BCCs. ${ }^{\mathrm{I}, \mathrm{I} 2}$

RHOGDIs (Rho GDP-dissociation inhibitors) make up a small group of proteins that negatively control RhoGTPases, which play important roles in cancer and metastasis. ${ }^{\text {I3 }}$ RHOGDI2 acts as an MSP in bladder tumors and probably in other types of epithelial tumors. ${ }^{\text {I }}$ However, RHOGDI2 may have cancer or tissue-specific functions in tumor suppression, while it might promote cancer invasiveness in a minority of human cancers. ${ }^{\text {I }, \mathrm{I} 5}$

The CD82/KAII protein, also called TSPAN27, is a member of the tetra-spantin family, which has important roles in adhesion, motility, and tumor progression. ${ }^{\text {I6, I7 }}$ It was initially demonstrated as an MSP in prostate carcinoma. ${ }^{\text {I8 }}$ The prognostic importance of this protein was then demonstrated in other human cancer types. ${ }^{17,19,20}$

Mitogen-activated protein kinase kinase $4\left(\mathrm{MKK}_{4}\right)$ is a component of MAP kinase in stress-activated protein kinase signaling. ${ }^{2,22}$ It was identified as an MSP in prostate and ovarian cancer. ${ }^{23,24}$ Although the tumor or metastasis suppressor function of $\mathrm{MKK}_{4}$ is generally accepted, there are also some clues that it has pro-oncogenic roles. ${ }^{22}$

AKAPı 2, also called SSeCKS/Gravin, is a scaffold protein and functions as a binding partner of protein kinase $\mathrm{C}$ and A, calmodulin, F-actin, cyclins, Src, and phospholipids. $^{25}$ Significant clinical and experimental evidence has shown that AKAPI2 is an important tumor and metastasis suppressor. ${ }^{25}$ AKAPI 2 expression is downregulated in various solid human cancers and some leukemias. ${ }^{25,26}$

The significance of MSPs in BCC is not well known due to the limited number of studies (Table I). Our aim was to demonstrate the distribution and expression of the seven important MSPs in BCC. We also tried to determine the relationship between protein expression levels, p53 status, and well-known clinicopathological parameters.

\section{Materials and methods}

\section{Study group}

A total of 96 BCCs from 92 patients (47 male/45 female) were included in this study. All patients were Caucasian, and the mean age was $66.3 \pm 13.4$ years. All lesions were excised from the head and neck area except for five lesions from the trunk. Normal epidermis samples adjacent to the BCCs (NE-BCC) and 10 nonlesional, histopathologically confirmed normal skin tissues $(\mathrm{N})$ were also studied. As there is no easy way to subclassify BCCs because of their wide and heterogeneous morphological spectrum, we used the criteria summarized by Carr et al. for classifying our group. ${ }^{27}$ Two major tumor groups were created and immunohistochemically scored to establish the differential expression patterns and contribution of the proteins:
1 Morphologically non-aggressive BCC types, including nodular, adenoid, superficial, and mixed carcinomas with less than $50 \%$ infiltrative pattern $(n=68)$.

2 Morphologically aggressive BCC types, including infiltrative BCCs with/without desmoplasia and mixed carcinomas with more than $50 \%$ infiltrative pattern $(n=28)$.

\section{Clinicopathological features}

The conventional clinicopathological parameters, including maximum diameter of the tumor, invasion depth, perineural invasion, anatomical invasion (Clark's) level, and local recurrences, were investigated. Tumor-associated inflammation was graded as previously described by Kaur et al. ${ }^{28}$

\section{Immunohistochemistry}

The classical, labeled streptavidin-biotin immunohistochemistry technique (UltraVision/DAB-Thermo Scientific, Waltham, MA, USA) was used for immunostaining of the slides. All steps of immunostaining were carried out by specific capillary coverplate technology in a Thermo-Shandon Sequenza ${ }^{\circledR}$ (Waltham, MA, USA) manual staining device. The negative control was performed by skipping the primary antibody step. Vendors, incubation time, antigen retrieval solutions, and positive controls are demonstrated in Table 2.

\section{Immunohistochemical analyses}

The immunohistochemistry results were analyzed semiquantitatively by using an immunohistochemical histological score (HSCORE) that included both the intensity and proportional distribution of specific staining. Based on a specific method described by McCarty et al., the HSCORE has been formulated as $\mathrm{HS}=\sum\left(P_{\mathrm{i}} \times i / 100\right)$, where $P_{\mathrm{i}}$ indicates the percentage of stained cells $(0-100 \%)$ at each intensity and $i$ shows the intensity of staining and ranges from no staining (0 points) to strong staining (3 points). ${ }^{29}$ The calculated HSCOREs were between 0 (no staining) and 300 (strong-diffuse staining) points. All calculations were performed with the Microsoft Office Excel ${ }^{\circledR}$ program using a simple macro. After evaluating the whole slide for specific staining, a minimum of five to seven randomly selected areas at medium power magnification $(\times 20)$ in normal and neoplastic tissues were analyzed for HSCORE. Nuclear (nuc) and cytoplasmic (cyt) expressions were evaluated separately for NM23-H1, MKK4, RHOGDI2, and NDRG1. Only membranocytoplasmic staining of E-cadherin, AKAP12, and CD82, and nuclear staining of p53 were accepted as positive.

\section{Statistical analysis}

Statistical analyses were performed using the PASW ${ }^{\circledR}$ Statistics 18 software (Chicago, IL, USA). The "Bonferroni correction" was applied for reducing the false-positive results. The differences between the HSCOREs of the groups were studied 


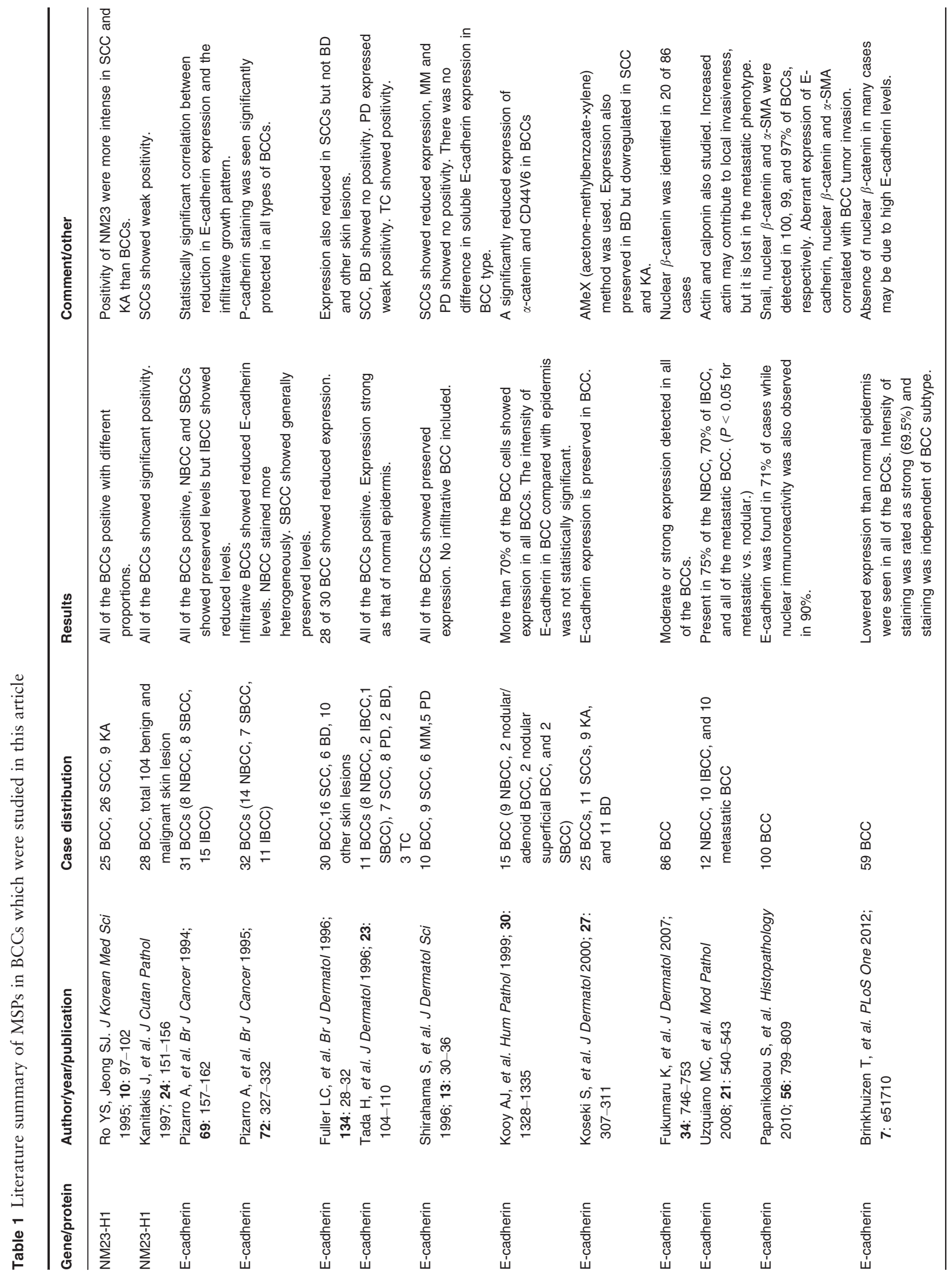




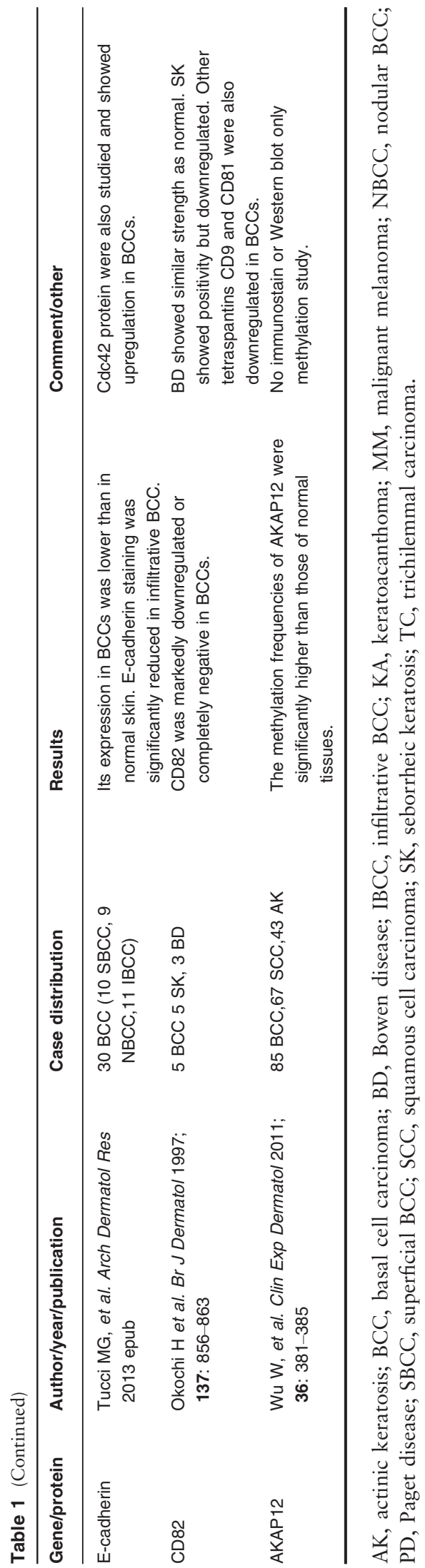

with the non-parametric Mann-Whitney $U$ test. The correlation between the parameters was investigated by Spearman's correlation test, and $r \geq 0.25$ and $P \leq 0.05$ were accepted as a significant correlation.

\section{Quantitative real-time polymerase chain reaction study group}

Quantitative real-time polymerase chain reaction (qRT-PCR) experiments were performed for frozen tissue consisting of 11 BCCs, three normal non-lesional skins, and eight normal skins adjacent to the BCCs. All tissues were re-confirmed by frozen sections before RNA isolation.

\section{Quantitative real-time polymerase chain reaction}

Total RNA was isolated from tissues using commercial RNA extraction kit (Fibrous Tissue kit; Qiagen, Hilden, Germany) in accordance with the manufacturer's instructions. A total of $500 \mathrm{ng}$ of total RNA was reverse-transcribed using oligo-dT primers for CDNA synthesis. qRT-PCR experiments were performed using the SYBR ${ }^{\circledR}$ Green chemistry in 96-well reaction plates with optical caps (Bioplastics, Landgraaf, Netherland) in an MX3005P (Strategene ${ }^{\circledR}$-Agilent, Santa Clara, CA, USA) thermocycler. GAPDH (glyceraldehyde-3-phosphate dehydrogenase) and HPRT1 (hypoxanthine phosphoribosyltransferase 1) genes were selected as reference genes. The qRT-PCR reaction contained $10 \mu \mathrm{l} 2 \times$ SYBR Green PCR Master Mix (Finnzyme-Thermo, Waltham, MA, USA), forward and reverse primers at optimized concentrations of $300 \mathrm{~nm}$ (150 nм for NDRG1 primers, $200 \mathrm{~nm}$ for MKK4 primers), $2 \mu \mathrm{l}$ cDNA template $(500 \mathrm{ng} / \mathrm{ml})$, and PCR grade water up to a final volume of $20 \mu \mathrm{l}$. The fluorescence data were also confirmed by meltcurve analysis for the specificity of the product. The primers used in this study are documented in Table 3.

\section{Quantitative real-time polymerase chain reaction data analysis}

Data analysis was performed with the free-use REST $\subset 2009$ (Qiagen) software, which gives reliable results in small study groups. ${ }^{30}$ This software uses the classic formulation

Ratio $=\left(E_{\text {target }}\right)^{\Delta \mathrm{CP}}$ target (control - sample) $\left./ E_{\text {ref }}\right)^{\Delta \mathrm{CP}}$ ref (control - sample) where $E$ is the amplification efficiency of the primers and CP is the cycle threshold, and shows both fold changes and standard errors between the controls and the samples. Statistical significance between the groups was evaluated by the Pair Wise Fixed Reallocation Randomization Test $\odot$ using the REST software.

\section{Results}

Immunohistochemical staining

In normal epidermis (N), all of the proteins were expressed at various intensities. NDRGI, E-cadherin, RHOGDI2, and cytoplasmic NM23-HI positivity was 
Table 2 Primer antibodies used in this study

\begin{tabular}{|c|c|c|c|c|c|}
\hline Antibody & Vendor & Dilution & Antigen retrieval & Incubation & Control tissue \\
\hline RHOGDI2 & Abcam & $1 / 100$ & Citrat; pH 6 & Overnight & Tonsil \\
\hline NM23-H1 & Abcam & $1 / 200$ & No & Overnight & Ductal carcinoma, breast \\
\hline MKK4 & Novocastra; Leica & $1 / 20$ & Citrat; pH 6 & Overnight & Ductal carcinoma, breast \\
\hline CD82 & Novocastra; Leica & $1 / 20$ & Citrat; pH 6 & Overnight & Tonsil \\
\hline AKAP12 & Atlas & $1 / 100$ & Citrat; pH 6 & Overnight & Testis \\
\hline NDRG1 & Santa Cruz & $1 / 100$ & EDTA; pH 9 & Overnight & Placenta \\
\hline E-cadherin & Cell Signaling & $1 / 100$ & Citrat; pH 6 & Overnight & Adenocarcinoma, colon \\
\hline P53 & Thermo & $1 / 100$ & Citrat; pH 6 & Overnight & Adenocarcinoma, colon \\
\hline
\end{tabular}

Table 3 qRT-PCR primer sequences used in this study

\begin{tabular}{|c|c|c|}
\hline Gene & $\mathbf{F}$ & $\mathbf{R}$ \\
\hline GAPDH & 5'-AGGTGAAGGTCGGAGTCAAC-3'a & 5'-GGGTCATTGATGGCAACA-3' \\
\hline$H P R T 1^{\mathrm{b}}$ & 5'-GCTGACCTGCTGGATTACAT-3' & 5'-CCCTGTTGACTGGTCATTAC-3' \\
\hline$K A / 1 / C D 82$ & 5'-AGCAGAACCCGCAGAGTCCT-3' & 5'-CTTCCACGAAACCAGTGCAG-3' \\
\hline$M A P 2 K 4^{c}$ & 5'-AGTGGACAGCTTGTGGACTCT-3 & 5'-AACTCCAGACATCAGAGCGGA-3' \\
\hline NM23 (NME1) & 5'-CCTGAAGGACCGTCCATTCT-3' & 5'-CCGTCTTCACCACATTCAGC-3' \\
\hline E-cadherin (CDH1) & 5'-GTCCTGGGCAGAGTGAATTT-3' & 5'-TCTGTGCCCACTTTGAATCG-3' \\
\hline AKAP12 & 5'-TCACAGAGGTTGGACAGAGA-3' & 5'-GTGAACAACCGCTGACTTAG-3' \\
\hline RHOGDI2 (ARHGDIB) & 5'-CCTCCACCACAGAAGTCCCT-3' & 5'-GCTTTCGGATCTGTCACCAC-3' \\
\hline NDRG1 & 5'-CAAGATCTCAGGATGGACC-3' & 5'-GACCACTTCCACGTTACTC-3' \\
\hline
\end{tabular}

${ }^{\mathrm{a}} \mathrm{Mol}$ Cancer 2010; 9: 226

${ }^{b}$ Designed by Dedeoglu BG, PHD.

${ }^{\mathrm{c}}$ Gynecol Oncol 2007; 105: 3 I 2-320.

strong and easily detectable. However, nuclear NM23-HI was only seen in the basal layers of the epidermis. AKAPr2, CD82/KAI, and cytoplasmic $\mathrm{MKK}_{4}$ were stained at medium intensities. Nuclear staining of $\mathrm{MKK}_{4}$ was very weak and not easily detectable. NE-BCC showed more heterogeneous positivity with all antibodies when compared to the normal epidermis.

In BCCs, both cytoplasmic (NDRGI $I_{\text {cyt }}$ ) and nuclear NDRGI (NDRG $I_{\text {nuc }}$ ) positivity were homogeneous (Fig. 2c, d). NDRGI $I_{\text {cyt }}$ was seen in all BCCs. However, only 74 of $96(77 \%)$ BCCs showed nuclear positivity. Similarly, NM23-Hi cytoplasmic expression (NM23-Hicyt) was also strong and diffused, except in two BCCs $(97.9 \%)$ (Fig. 2a, b). Nuclear expression of NM23-HI was weaker and expressed in 73 of $96(76 \%)$ cases. ECadherin antibody was represented by both membranous and cytoplasmic positivity except in seven BCCs (92.7\%) (Fig. 2e, f). Nuclear staining was seen very rarely and usually in strongly stained areas. CD82/KAI and AKAPI 2 positivity was significantly reduced and only seen in focal areas of the BCCs in I4 (I5.I\%) and $2 \mathrm{I}$ of 96 (2I.8\%) cases, respectively (Fig. $3 \mathrm{C}-\mathrm{f}$ ). $\mathrm{MKK}_{4}$ immunostaining of neoplastic tissues was weak/medium cytoplasmic positive in $73(76 \%)$ and weak nuclear positive in only $35(36.4 \%)$ cases (Fig. 3a,b). The nuclear expression was more evident in normal tissues. Although RHOGDI2 staining showed cytoplasmic positivity (RHOGDI $2_{\text {cyt }}$ ) in 89 of $96(92.7 \%)$ cases, the intensity was significantly reduced (Fig. 2g,h). Nuclear expression (RHOGDI $2_{\text {nuc }}$ ) was very weak and heterogeneously in 59 (6I.4\%) of 96 BCCs.

\section{HSCORES}

The HCORES of the groups are demonstrated in the boxplot graph (Fig. I).

\section{Statistical results}

As the tumor microenvironment changes are part of carcinogenesis, we evaluated the difference between the NEBCC and normal non-lesional (N) skin. Although there was no statistical difference for $\mathrm{RHOGDI}_{2}$ cyt $\mathrm{NM}_{23}$ $\mathrm{HI}_{\text {cyt/nuc }}$, CD82, and $\mathrm{MKK}_{4}$ cyt/nuc scores between the two groups, the other markers showed a significant reduction in NE-BCC $(P \leq 0.05)$.

When normal epidermis was compared to BCCs, all of the scores except for $\mathrm{NM} 23$ cyt/nuc were significantly lower in the tumor groups $(P \leq \mathrm{O} . \mathrm{OI})$. Similarly, when NE-BCC was compared to BCCs, all of the markers except for $\mathrm{NM}_{23}-\mathrm{HI}_{\text {cyt }}$ and $\mathrm{NDRGI}_{\text {cyt }}$ indicated significantly reduced scores $(P \leq \mathrm{O} . \mathrm{OI})$ in the BCC group. Furthermore, $\mathrm{NM} 23-\mathrm{HI}_{\text {nuc }}$ scores were higher in BCCs than in NE-BCC. 
(a)

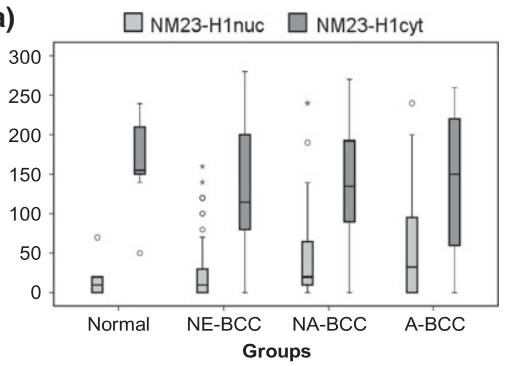

(c)

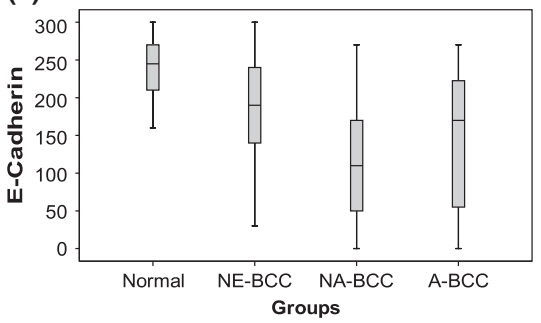

(e)

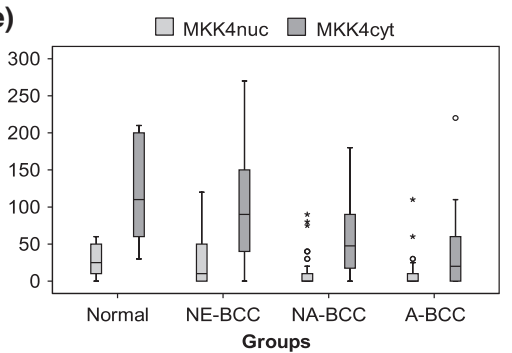

(g)

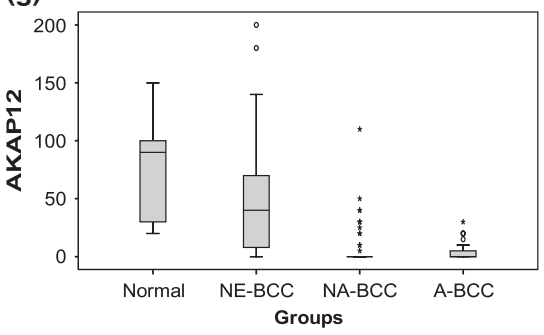

(b)

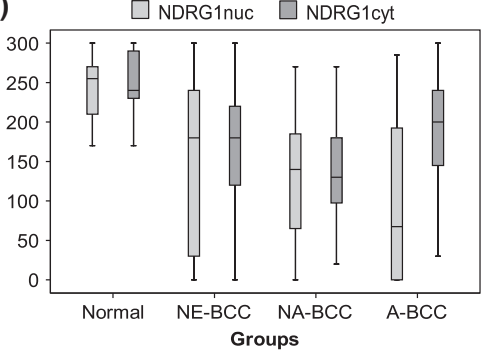

(d)

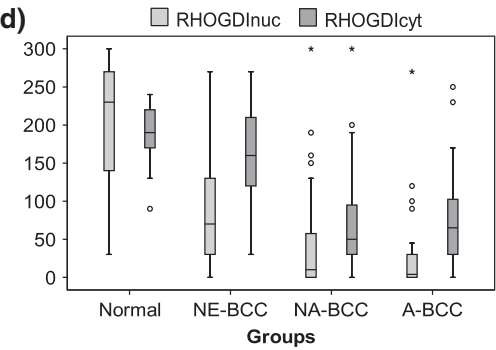

(f)

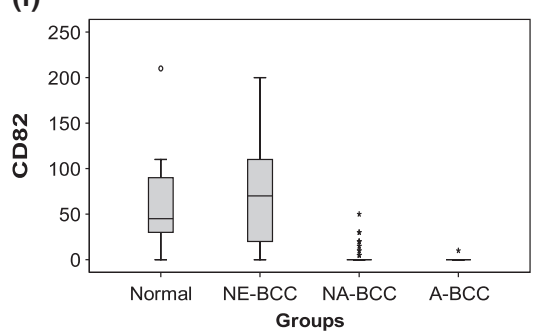

Figure 1 Boxplot graphics of the groups. (a) $\mathrm{NM}_{23}-\mathrm{HI}$; (b) NDRGI; (c) E-cadherin; (d) RHOGDI2; (e) $\mathrm{MKK}_{4}$; (f) CD82; (g) AKAPı 2. The protected levels of NM23-Hi (a), NDRGI (b) and E-cadherin (c) in BCCs are clearly demonstrated in boxplot graphics. Conversely, significant downregulation or lost of CD82 (f) and AKAPI 2 (g) HSCOREs have attracted attention in the tumor group. For RHOGDI2 (d) and $\mathrm{MKK}_{4}$ (e) HSCOREs, although the downregulation is seen in both nuclear and cytoplasmic scores, reduction of nuclear HSCOREs are more significant. A-BCC, aggressive BCC; BCC, basal cell carcinoma; NABCC, non-aggressive BCC; NE-BCC, normal epidermis adjacent to BCC. * and ${ }^{\circ}$ demonstrate more than one case with similar HSCORE
Morphologically aggressive BCCs expressed significantly higher $\mathrm{NDRGI}_{\mathrm{cyt}}$ scores $(P=0.00 \mathrm{I})$ and lower CD82/KAII scores $(P=0.048)$.

BCCs with perineural invasion showed lower nuclear NM23-HI levels $(P=$ o.OI $)$. Recurrent BCCs expressed higher RHOGDI $2_{\text {nuc }}$ levels $(P=\mathrm{O} . \mathrm{OI})$ when compared to the non-recurrence group.

\section{Correlation analysis}

In the BCC group, there were significant correlations $(P=$ O.OI level) between several markers as follows: NM23$\mathrm{HI}_{\text {nuc }}-\mathrm{NM}_{23}-\mathrm{HI}_{\mathrm{cyt}} \quad(r=0.442) ; \quad$ AKAPI $2-\mathrm{RHOGDI}_{\mathrm{cyt}}$ $(r=0.333)$; ${\text { AKAPI } 2-N D R G I_{c y t}}(r=0.280)$; E-cadherin$\mathrm{RHOGDI}_{\text {cyt }} \quad(r=0.303) ;$ E-cadherin-NDRGI $\mathrm{I}_{\mathrm{cyt}} \quad(r=$ $0.4 \mathrm{I} 3) ; \mathrm{RHOGDI}_{2} \mathbf{n c}_{\mathrm{nu}}-\mathrm{RHOGDI}_{\mathrm{cyt}}(r=0.405)$; RHO$\mathrm{GDI}_{2}$ nuc $_{\text {nKK }}-\mathrm{MKyt}_{4} \quad(r=-0.294) ; \mathrm{NDRG}_{\text {nuc }}-\mathrm{NDRGI}_{\mathrm{cyt}}$ $(r=0.356) ;$ and $\mathrm{MKK}_{4 \text { nuc }}-\mathrm{MKK}_{4 \mathrm{cyt}}(r=0.365)$. There were also significant negative correlations between AKAPI 2 and inflammation $(r=-0.275 ; P=0.007)$.

A randomly selected subgroup of 44 BCCs from the main group was stained with p $53_{3}$ primary antibody to establish the correlation between MSPs and $\mathrm{p}_{53}$. We detected only a negative correlation with RHOGDI $2_{\text {cyt }}$ $(r=-0.3$ I $6 ; P=0.037)$.

\section{Relative expression software tool (REST) analysis of quantitative real-time polymerase chain reaction data}

BCCs were compared to both the normal non-lesional skin tissue and the skin adjacent to neoplasia groups. We found significant upregulation of NM23 (I.4-fold, $P=0.032$ ) and downregulation of $A K A P_{I 2}$ (-I.2-fold; $P=0.006)$ when BCC was compared to normal skin. 
Figure 2 Significant $\mathrm{NM}_{23}-\mathrm{H}_{\mathrm{I}}$ positivity is seen in both nodular (a) and infiltrative basal cell carcinomas (BCCs). (b). NM23-HI immunostaining highlights basal palisading cells in nodular type BCC. (c,d) Similar to NM23, strong NDRGI expression is detected in BCCs. (e,f) E-cadherin expression is protected in BCCs. (g,h) RhoGDI expression is very weak or negative in BCCs. The contrast between positive inflammatory cells and carcinoma cells is clearly demonstrated $(\mathrm{h})$. (a,f, $\mathrm{h}, \times 200 ; \mathrm{b}-\mathrm{e}, \mathrm{g}, \times 100)$
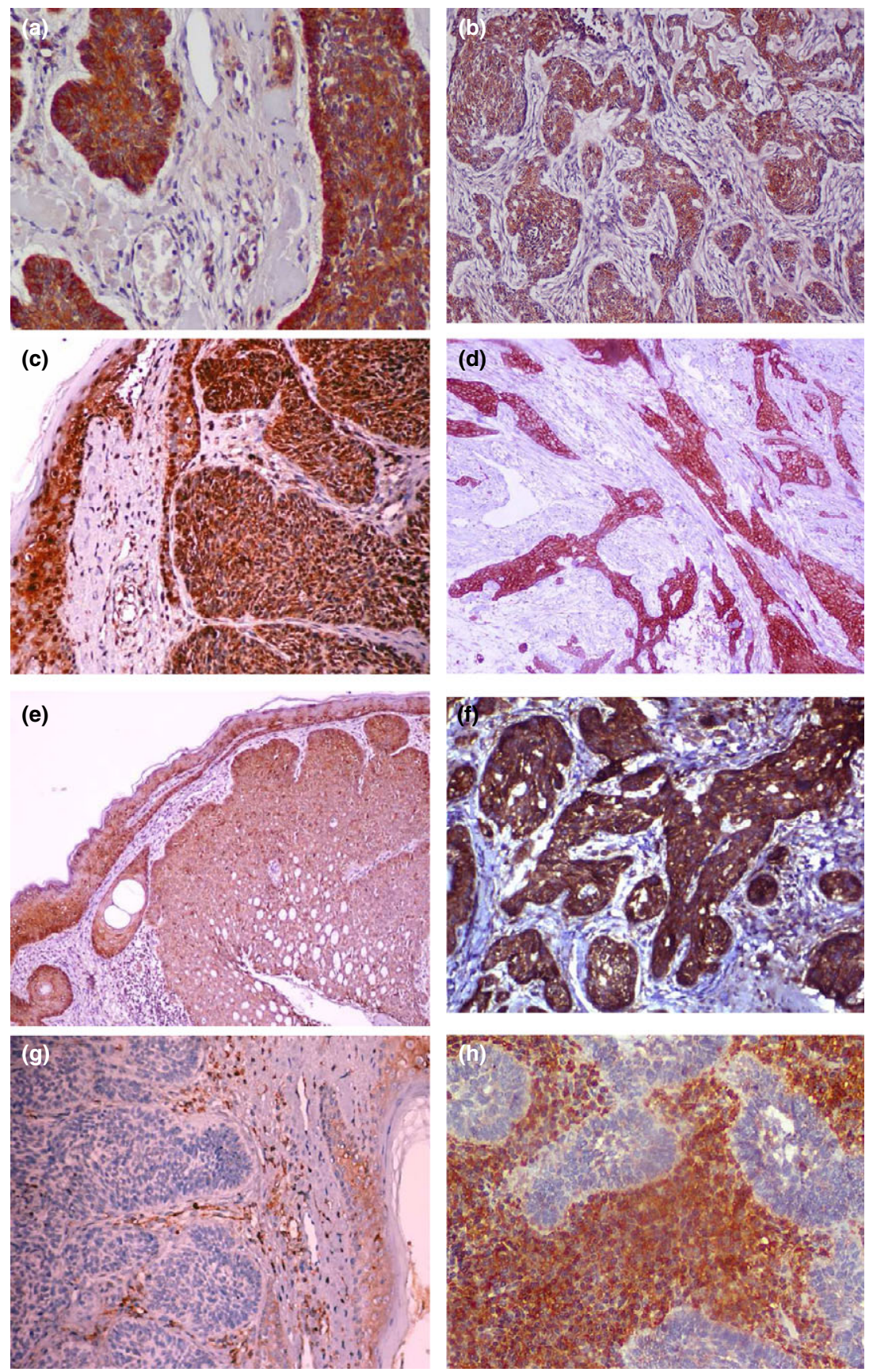

$N D R G_{I}$ showed statistically significantly higher levels (2.2-fold, $P=0.00 \mathrm{I})$ in BCC when compared to the skin adjacent to the neoplasia, similar to the immunohistochemical results.

\section{Discussion}

Invasion and metastasis are the most important hallmarks of cancer and well-known clinical signs of a poor prognosis. ${ }^{31,32}$ BCCs display all the hallmarks of cancer, including invasion, with the exception of metastasis. To date, the question of why BCCs metastasize only rarely has not been adequately answered. A possible but unsupported explanation for this question is the strict stromal dependence of BCC. ${ }^{33}$ In this study, we examined the contribution of the MSPs in the non-metastasizing feature of BCCs and focused on seven well-known proteins.

We detected that NM23-HI HSCOREs were protected in BCCs, and mRNA level of NM23-HI was higher in BCCs than in normal skin. Similarly, Ro and Jeong and 

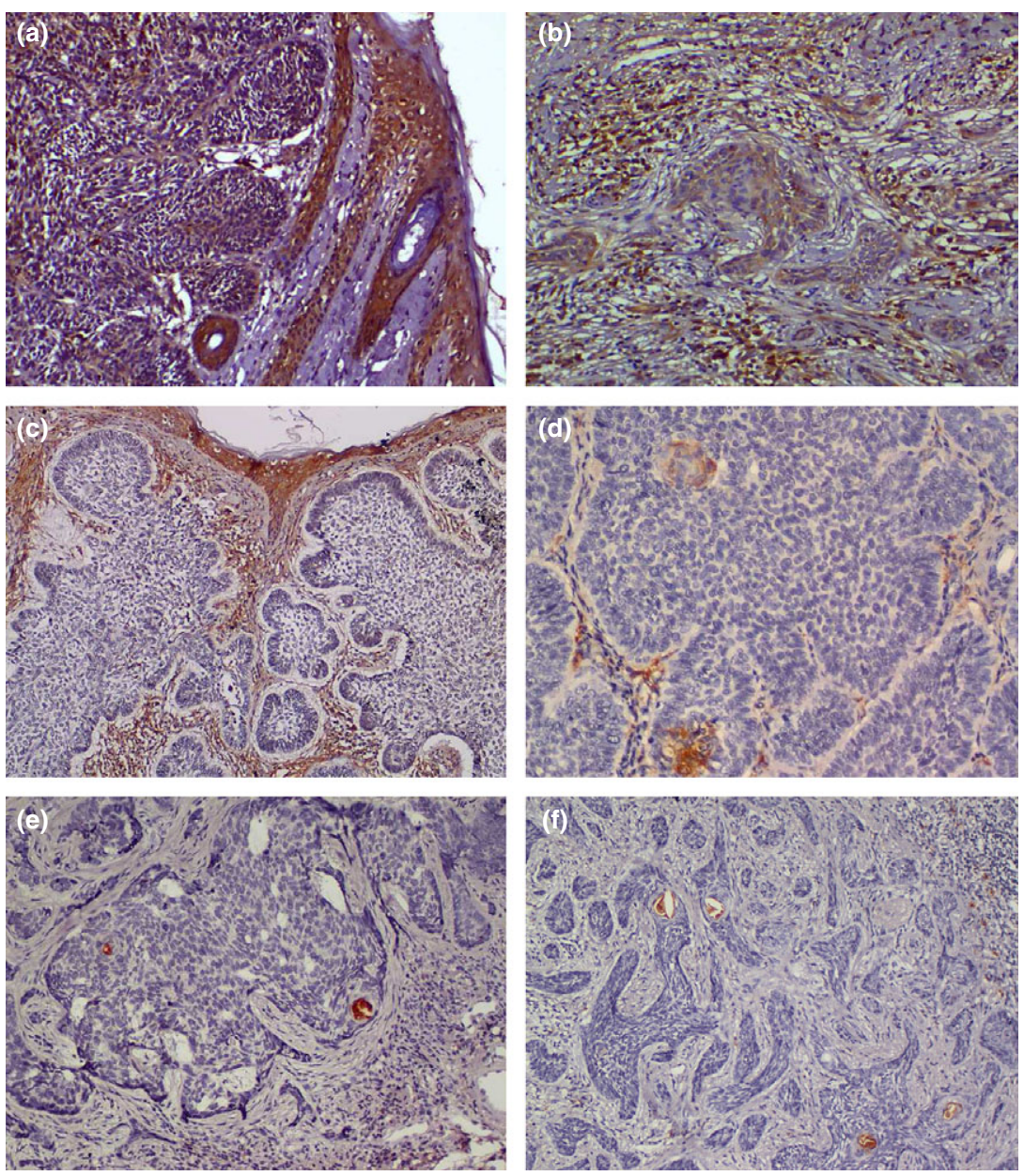

Figure 3 Normal skin adjacent to basal cell carcinoma (BCC) expresses medium strength nuclear and cytoplasmic $\mathrm{MKK}_{4}$ staining. Nodular (a) and infiltrative BCCs (b) show reduced nuclear and cytoplasmic staining. (c) AKAPı 2 expression is clearly seen in normal epidermis and stromal cells but not in BCCs. (d) Weak AKAPI 2 staining is detected only at squamous differentiation areas in a BCC case. Stromal cells are also positive in this case. CD82 expression is not detected in nodular (e) and infiltrative (f) BCCs except in focal squamous differentiation areas. (a,c,f, $\times$ IOO; b,d,e, $\times 200$ )
Kanitakis et al. focused on the $\mathrm{NM}_{2} 3-\mathrm{HI}$ protein in skin lesions and both found medium/strong NM23-HI positivity in all BCCs. ${ }^{34,35}$ In spite of the significant expression of $\mathrm{NM}_{23}-\mathrm{HI}$ in BCCs, its importance is not well known. However, it has been shown that $\mathrm{NM}_{23}-\mathrm{HI}_{\mathrm{I}}$ expression is inversely related to the metastasis status in other human carcinomas. ${ }^{36}$ The significant $\mathrm{NM}_{23}-\mathrm{HI}_{\mathrm{I}}$ expression in BCCs probably contributes to their non-metastasizing feature.

One of the important results of this study is the demonstration of significant cytoplasmic NDRGI expression in BCC, which is also supported with the qRT-PCR study. Although NDRGI expression has not been studied in BCC previously, Cangul demonstrated that human carcinomas expressed high levels of NDRGI compared to their normal counterpart, ${ }^{37}$ and the prognostic importance of NDRGI expression has been pointed out in various human tumors. ${ }^{38-4 \mathrm{I}}$ We also found a significant correlation between NDRGI $\mathrm{I}_{\text {cyt }}(P=0.00 \mathrm{I})$ and E-cadherin in BCCs, and this relationship was reported in the literature in colon and prostate carcinomas. ${ }^{8,42,43}$ Our data clearly support an E-cadherin/NDRGI pathway in human carcinomas.

Besides NM23-Hi and NDRGI, E-cadherin expression was generally preserved in $92.7 \%$ of BCCs with relatively higher HSCORES, and qRT-PCR studies also showed no statistically significant difference from normal skin tissue. The data from the literature and our study show that E-cadherin positivity is expected in BCC, even if at reduced levels compared to the normal epidermis (Table I).

CD82/KAII and AKAPI2 expressions were significantly reduced or completely lost in all morphological subtypes of BCCs. We also detected downregulation of AKAPI 2 mRNA in BCC. Similar to our results, the downregulation of CD82/KAII expression in BCCs was demonstrated before. ${ }^{44}$ Yet, the expression pattern of AKAPI 2 is still not known in BCCs, a recent article pointed out that the methylation frequencies of the $A K A P_{I 2}$ gene were significantly higher in skin carcinomas than normal skin tissue. ${ }^{45} \mathrm{CD} 82 / \mathrm{KAII}$ and AKAPI2 probably have no contribution to the non-metastatic features of BCCs. 
However, we believe that these proteins are interesting negative markers for BCCs, and further studies might show their role in the differential diagnosis.

One of the goals of the study was to show the correlation between the MSPs, and important clinicopathological parameters and $\mathrm{p}_{53}$ in BCCs. We found AKAPI2 inversely correlated with inflammation, together with an inverse relationship between $\mathrm{NM}_{2} 3-\mathrm{HI}_{\text {nuc }}$ and perineural invasion. Besides these expected correlations, we found that recurrences were correlated only with RHOGDI $\boldsymbol{2}_{\text {nuc }}$. This result may be explained by the dual and unpredicted role of RHOGDI2 in carcinomas as proposed by Griner and Theodorescu. ${ }^{\mathrm{I}}{ }^{4} \mathrm{We}$ found only an inverse correlation between RHOGDI $2_{\text {cyt }}$ and p53. Although the relationship between $\mathrm{p}_{5} 3$ and RHOGDI2 has not been demonstrated previously, interaction between $\mathrm{p}_{53}$ and $\mathrm{CD} 82 / \mathrm{KAII}$, another MSP, has been reported. ${ }^{4-48}$ However, other studies have been querying this correlation, similar to our results. ${ }^{49^{-52}}$

One of the major questions in this study is the contribution of MSPs to the aggressive phenotype of BCCs. We detected upregulation of NDRGI levels in the aggressive phenotype $(P=0.00 \mathrm{I})$. Similarly, CD82/KAI levels $(P=$ $0.048)$ were downregulated. In the literature, E-cadherin levels have been shown to be downregulated in aggressive BCCs, ${ }^{\mathrm{I}, 53}$ but this has not been supported by other studies (Table I). These results may show a slightly different profile of MSPs in aggressive carcinomas than non-aggressive BCCs.

In conclusion, we have demonstrated differential expression patterns for the seven MSPs in BCCs. AKAPı 2 and CD82/KAII levels were significantly reduced in BCCs. However, NM23-Hi, NDRGi, and E-cadherin levels were minimally reduced, and they were generally expressed in this neoplasm group. The other markers, $\mathrm{MKK}_{4}$ and RHOGDI2, were also reduced but not lost in BCCs. Although this is a very simplified approach, preserved levels of NM23-HI, E-cadherin, and NDRGI may contribute to the non-metastatic features of BCCs. One of our important findings is there are plenty of significant correlations among the MSPs. Data from this study might reveal possible pathways between MSPs, when combined with the current knowledge on pathways. This relationship between these MSPs warrants further biological and experimental pathway research.

\section{Acknowledgments}

This study was supported by the Scientific and Technical Research Council of Turkey (TUBITAK) (grant no. SBAG-IO8SI 84). The project was approved by the Local Ethical Committee - Krrıkkale (07.04.2008/ 2008-039).

\section{References}

I Ting PT, Kasper R, Arlette JP. Metastatic basal cell carcinoma: report of two cases and literature review. J Cutan Med Surg 2005; 9: IO-I 5.

2 Varga E, Korom I, Rasko Z, et al. Neglected basal cell carcinomas in the 2Ist century. J Skin Cancer 20II; 2OII: 392I 5 I.

3 Cook LM, Hurst DR, Welch DR. Metastasis suppressors and the tumor microenvironment. Semin Cancer Biol 2OII; 2I: II3-I22.

4 Novak M, Jarrett SG, McCorkle JR, et al. Multiple mechanisms underlie metastasis suppressor function of NM23-Hi in melanoma. Naunyn Schmiedebergs Arch Pharmacol 20II; 384: 433-438.

5 Horak CE, Mendoza A, Vega-Valle E, et al. Nm23-HI suppresses metastasis by inhibiting expression of the lysophosphatidic acid receptor EDG2. Cancer Res 2007; 67: II75 I-II759.

6 Kovacevic Z, Richardson DR. The metastasis suppressor, Ndrg-I: a new ally in the fight against cancer.

Carcinogenesis 2006; 27: 2355-2366.

7 Kitowska A, Pawelczyk T. N-myc downstream regulated I gene and its place in the cellular machinery. Acta Biochim Pol 2010; 57: I 5-2I.

8 Guan RJ, Ford HL, Fu Y, et al. Drg-I as a differentiation-related, putative metastatic suppressor gene in human colon cancer. Cancer Res 2000; 60: 749-755.

9 Schmalhofer O, Brabletz S, Brabletz T. E-cadherin, betacatenin, and $\mathrm{ZEBI}$ in malignant progression of cancer. Cancer Metastasis Rev 2009; 28: I 5 I-I66.

Io Jeanes A, Gottardi CJ, Yap AS. Cadherins and cancer: how does cadherin dysfunction promote tumor progression? Oncogene 2008; 27: 6920-6929.

I I Pizarro A. E-cadherin expression is frequently reduced in infiltrative basal cell carcinoma. J Dermatol 2000; 27: 804-805.

I2 Papadavid E, Pignatelli M, Zakynthinos S, et al. Abnormal immunoreactivity of the E-cadherin/catenin (alpha-, beta-, and gamma-) complex in premalignant and malignant non-melanocytic skin tumours. J Pathol 2002; I96: I 54-I62.

I3 DerMardirossian C, Bokoch GM. GDIs: central regulatory molecules in Rho GTPase activation. Trends Cell Biol 2005; I5: 356-363.

I4 Griner EM, Theodorescu D. The faces and friends of RhoGDI2. Cancer Metastasis Rev 20I2; 3I: 5I9-528.

I 5 Zhang Y, Zhang B. D4-GDI, a Rho GTPase regulator, promotes breast cancer cell invasiveness. Cancer Res 2006; 66: 5592-5598.

I 6 Bassani S, Cingolani LA. Tetraspanins: interactions and interplay with integrins. Int J Biochem Cell Biol 20I2; 44: 703-708.

I7 Romanska HM, Berditchevski F. Tetraspanins in human epithelial malignancies. J Pathol 20I I; 223: 4-I4.

I 8 Dong JT, Suzuki H, Pin SS, et al. Down-regulation of the KAII metastasis suppressor gene during the progression 
of human prostatic cancer infrequently involves gene mutation or allelic loss. Cancer Res I996; 56: $4387^{-}$ 4390.

I9 Yang X, Wei L, Tang C, et al. KAII protein is downregulated during the progression of human breast cancer. Clin Cancer Res 2000; 6: 3424-3429.

20 Christgen M, Bruchhardt H, Ballmaier M, et al. KAII/ $\mathrm{CD} 82$ is a novel target of estrogen receptor-mediated gene repression and downregulated in primary human breast cancer. Int J Cancer 2008; I23: 2239-2346.

2I Knopeke MT, Ritschdorff ET, Clark R, et al. Building on the foundation of daring hypotheses: using the $\mathrm{MKK}_{4}$ metastasis suppressor to develop models of dormancy and metastatic colonization. FEBS Lett 20I I 585: 3 I 593165 .

22 Whitmarsh AJ, Davis RJ. Role of mitogen-activated protein kinase kinase 4 in cancer. Oncogene 2007; 26: 3I $72-3$ I 84

23 Yoshida BA, Dubauskas Z, Chekmareva MA, et al. Mitogen-activated protein kinase kinase $4 /$ stress-activated protein/Erk kinase I $\left(\mathrm{MKK}_{4} / \mathrm{SEK}_{\mathrm{I}}\right)$, a prostate cancer metastasis suppressor gene encoded by human chromosome I7. Cancer Res I999; 59: 5483-5487.

24 Yamada SD, Hickson JA, Hrobowski Y, et al. Mitogenactivated protein kinase kinase $4\left(\mathrm{MKK}_{4}\right)$ acts as a metastasis suppressor gene in human ovarian carcinoma. Cancer Res 2002; 62: 6717-6723.

25 Gelman IH. Suppression of tumor and metastasis progression through the scaffolding functions of SSeCKS/ Gravin/AKAPI 2. Cancer Metastasis Rev 20I2; 3I: 493500.

26 Gelman IH. Emerging roles for SSeCKS/Gravin/AKAPı 2 in the control of cell proliferation, cancer malignancy, and barriergenesis. Genes Cancer 2010; I: I I 47-I I 56 .

27 Carr RA, Taibjee SM, Sanders DSA. Basaloid skin tumours: basal cell carcinoma. Curr Diagn Pathol 2007; I3: $252-272$.

28 Kaur P, Mulvaney M, Carlson JA. Basal cell carcinoma progression correlates with host immune response and stromal alterations: a histologic analysis. Am J

Dermatopathol 2006; 28: 293-307.

29 McCarty KS, Szabo E, Flowers JL, et al. Use of a monoclonal anti-estrogen receptor antibody in the immunohistochemical evaluation of human tumors. Cancer Res I986; 46: 4244s-4248s.

30 Pfaffl MW, Horgan G, Dempfle L. Relative expression software tool (RESTC) for group-wise comparison and statistical analysis of relative expression results in realtime PCR. Nucleic Acids Res 2002; 30 : e36.

3 I Hanahan D, Weinberg RA. Hallmarks of cancer: the next generation. Cell 20II; I44: 646-674.

32 Leber MF, Efferth T. Molecular principles of cancer invasion and metastasis (review). Int J Oncol 2009; 34: $88 \mathrm{I}-895$.

33 Blewitt RW. Why does basal cell carcinoma metastasize so rarely? Int J Dermatol I980; I9: I44-I46.
34 Ro YS, Jeong SJ. Expression of the nucleoside diphosphate kinase in human skin cancers: an immunohistochemical study. J Korean Med Sci I995; Io: 97-IO2.

35 Kanitakis J, Euvrard S, Bourchany D, et al. Expression of the nm23 metastasis-suppressor gene product in skin tumors. J Cutan Pathol I997; 24: I 5 I-I 56.

36 Tee YT, Chen GD, Lin LY, et al. Nm23-Hi: a metastasis-associated gene. Taiwan J Obstet Gynecol 2006; 45: IO7-II3.

37 Cangul H. Hypoxia upregulates the expression of the NDRGI gene leading to its overexpression in various human cancers. BMC Genet 2004; 5: 27.

38 Bandyopadhyay S, Wang Y, Zhan R, et al. The tumor metastasis suppressor gene Drg-I down-regulates the expression of activating transcription factor 3 in prostate cancer. Cancer Res 2006; 66: II983-II990.

39 Bandyopadhyay S, Pai SK, Hirota S, et al. Role of the putative tumor metastasis suppressor gene Drg-I in breast cancer progression. Oncogene 2004; 23: 5675-568I.

40 Strzelczyk B, Szulc A, Rzepko R, et al. Identification of high-risk stage II colorectal tumors by combined analysis of the NDRGI gene expression and the depth of tumor invasion. Ann Surg Oncol 2009; I6: I 287-I294.

4I Chua MS, Sun H, Cheung ST, et al. Overexpression of NDRGI is an indicator of poor prognosis in hepatocellular carcinoma. Mod Pathol 2007; 20: 76-83.

42 Kachhap SK, Faith D, Qian DZ, et al. The N-Myc down regulated Gene I (NDRGI) Is a Rab4a effector involved in vesicular recycling of E-cadherin. PLOS ONE 2007; 2: e844.

43 Song Y, Oda Y, Hori M, et al. N-myc downstream regulated gene-I/Cap43 may play an important role in malignant progression of prostate cancer, in its close association with E-cadherin. Hum Pathol 20IO; 4I: 214222.

44 Okochi H, Kato M, Nashiro K, et al. Expression of tetra-spans transmembrane family (CD9, $\mathrm{CD}_{37}, \mathrm{CD}_{53}$, $\mathrm{CD}_{3}, \mathrm{CD} 8 \mathrm{I}$ and $\mathrm{CD} 82$ ) in normal and neoplastic human keratinocytes: an association of $\mathrm{CD}_{9}$ with alpha 3 beta I integrin. Br J Dermatol I997; I37: $856-863$.

$45 \mathrm{Wu} \mathrm{W}$, Zhang J, Yang H, et al. Examination of AKAPI2 promoter methylation in skin cancer using methylationsensitive high-resolution melting analysis. Clin Exp Dermatol 20II; 36: 38I-385.

46 Mashimo T, Watabe M, Hirota S, et al. The expression of the KAIr gene, a tumor metastasis suppressor, is directly activated by p53. Proc Natl Acad Sci USA I998; 95: II307-II3II.

47 Guo C, Liu QG, Zhang L, et al. Expression and clinical significance of $\mathrm{p}_{53}$, JunB and KAII/CD82 in human hepatocellular carcinoma. Hepatobiliary Pancreat Dis Int 2009; 8: 389-396.

48 Marreiros A, Dudgeon K, Dao V, et al. KAII promoter activity is dependent on $\mathrm{p}_{53}$, junB and $\mathrm{AP}_{2}$ : evidence for a possible mechanism underlying loss of KAII expression in cancer cells. Oncogene 2004; 24: 637-649. 
49 Geradts J, Maynard R, Birrer MJ, et al. Frequent loss of KAII expression in squamous and lymphoid neoplasms. An immunohistochemical study of archival tissues. Am J Pathol I999; I54: I665-I67I.

50 Uzawa K, Ono K, Suzuki H, et al. High prevalence of decreased expression of KAII metastasis suppressor in human oral carcinogenesis. Clin Cancer Res 2002; 8: 828-835.

5I Miyazaki T, Kato H, Shitara Y, et al. Mutation and expression of the metastasis suppressor gene KAII in esophageal squamous cell carcinoma. Cancer 2000; 89: 955-962.

52 Jackson P, Ow K, Yardley G, et al. Downregulation of KAII mRNA in localised prostate cancer and its bony metastases does not correlate with p 53 overexpression. Prostate Cancer Prostatic Dis 2003; 6: I74-I 8 I.

53 Pizarro A, Benito N, Navarro P, et al. E-cadherin expression in basal cell carcinoma. Br J Cancer I994; 69: I $57-$ I62. 